\title{
Stock Price Behavior around Extreme Trading Volumes
}

\author{
Megan Y. Sun \\ College of Business and Economics, University of Wisconsin - River Falls \\ 410 S. Third Street, River Falls, WI 54022, USA
}

Tel: 1-715-425-3335Ｅ-mail: megan.sun@uwrf.edu

\author{
Received: January 7, 2013 \\ Accepted: January 24, 2013 \\ Online Published: January 24, 2013 \\ doi:10.5430/afr.v2n1p61 \\ URL: http://dx.doi.org/10.5430/afr.v2n1p61
}

\begin{abstract}
The study investigates how the coexistence of extreme returns and volumes predicts future stock returns and how the high-volume return premium is affected by the coexistent extreme returns. It also examines the patterns of returns, volatility, and skewness around extreme trading volumes. We find that stocks exhibit different return and volatility patterns prior to and after extreme volumes. We also find that the high-volume return premium only exists among small size stocks which simultaneously experience extremely low prior returns. The high-volume return premium disappears for larger size stocks experiencing extremely low prior returns. Regardless of the firm size, the high-volume return premium only lasts for a very short time period for stocks simultaneously experiencing extremely high prior returns. We document that while accompanied by extreme volumes, past losers do not outperform past winners, and past winners do not outperform past losers neither. The existence of extreme volumes cancels out any potential gains from contrarian or momentum investing strategies.
\end{abstract}

Keywords: Extreme trading volumes, Return reversal, Return continuation

\section{Introduction}

Finance literature suggests that past extreme events could affect investor's decisions and stock returns afterwards. One of the extreme events is volume extreme. Gervais, Kaniel and Mingelgrin (2001), Garfinkel and Sokobin (2006), Lerman, Livnat, and Mendenhall (2011), and Kanile, Ozoguz and Starks (2012) clearly document that stocks experiencing extremely high volumes tend to experience subsequent positive excess returns, while stocks experiencing extremely low volumes tend to experience negative excess returns not only in US but also in other developed and emerging equity markets. This phenomenon is called the high-volume return premium.

Just as much as extreme volumes affect stock returns, past extreme returns can affect investor's trading decisions too. Huddart, Lang, Yetman (2009) investigate the volume and price patterns around a stock's 52-week price highs and lows and find an elevated trading volume and positive excess returns following the extreme event, especially for small firms. George and Hwang (2004) study momentum investing strategy and recommend focusing on stocks that are traded in the top 30\% of the prior trading range. Driessen, Lin and Hemert (2011) find that the volatility of stock returns increases after stock price break through 52-week high or low. Clearly, price (return) extremes impact asset price behavior too.

Since both extreme returns and extreme volumes affect stock prices, it would be beneficial to investigate how past returns and past volume information simultaneously predict future returns. As Wang (1994) points out, incorporating volume information into asset pricing can substantially improve the predictability of returns. Conrad, Hameed, and Niden (1994) document that high volume securities experience price reversals, while low volume securities experience price continuations. Later, Cooper (1999) finds that decreasing-volume stocks experience greater reversals, while increasing-volume stocks exhibit weaker reversals and positive autocorrelation. However, none of the above research considers the interaction between extreme returns and extreme volumes. Given that extreme volumes in either direction could be accompanied by either extremely high returns or extremely low returns, this study focuses on how combining extreme returns and extreme volumes help predict future returns (Note 1). Traders on the stock market have long treated trading volume as precious information when evaluating the strength of the market. Conventional market wisdom holds that when prices are up and volume is rising, or when prices are down and volume is declining, the market is strong. By contrast, lower prices and rising volume, or higher prices and declining volume indicate a weak market. This study investigates these various scenarios, treating relationships between volume and returns in all their complexities, while attempting to clarify them. 
Another related issue our study focuses on is how stock returns behave around the extreme volumes. As finance literature, for instance, MacNeil, Frey, and Embrechts (2005) shows, obtaining information about the shape of the return distribution is very important in both theoretical and empirical analysis since theoretical models needs these information as parameters and statistical inference relies heavily on the distributional assumptions. Therefore, investigating how stock returns behave around the extreme volume events could shed more lights on this issue.

This study, then, contributes to finance literature in two ways. First, this study investigates returns, volatility, and skewness patterns around extreme trading volumes. We find that stocks experiencing extremely high volumes are often accompanied by higher prior and future returns when compared to stocks with extremely low volumes. Small stocks tend to earn more returns after extreme low volumes, but less returns after extreme high volumes, while larger stocks tend to earn less returns after any type of volume extremes, high or low. Small stock returns are more volatile, but less skewed before extremely low volumes than before extremely high ones, while larger size groups are more volatile and more skewed after extremely high volumes than after extremely low ones. Small stocks tend to become less volatile after experiencing extreme low volumes, while all stocks tend to become more volatile after experiencing extreme high volumes. These findings are significant because they provide a fuller account of how extreme volumes influence the volatility and skewness of future returns.

Second, we demonstrate how the coexistence of extreme volumes and extreme returns predicts future returns - a correlation not found in previous literature. After controlling for the level of extreme returns, we find that the high-volume return premium only exists among small size stocks which simultaneously experience extremely low prior returns, but not among larger size stocks experiencing similar low prior returns. The high-volume return premium does not hold long for stocks simultaneously experiencing extremely high prior returns regardless of size. When accompanied by extreme trading volumes, high or low, neither return reversals nor continuations can be observed. Therefore, the traditional contrarian strategy and momentum strategy don't have any effects on stocks simultaneously experiencing extreme volumes on either end.

The remainder of the study is organized into five more sections. Section two reviews prior finance literature related to our research. Section three provides the data used in our study. Section four describes the methodology employed. Section five presents the empirical results. Section six concludes our study with a summary of evidence.

\section{Previous Research}

Quite a few researches have contributed to our understanding of the high-volume return premium in recent years. Gervais, Kaniel, and Mingelgrin (2001) are among the first to document the existence of the high-volume return premium. They find that stocks experiencing extremely high trading volumes over a day- or week-long period tend to appreciate over the following months while those experiencing extremely low trading volumes will depreciate over the following months. They attribute this high-volume return premium to visibility hypothesis. Garfinkel and Sokobin (2006) provide evidence for a positive correlation between abnormal share trading volume in the three-day period around earnings announcement and abnormal returns over the subsequent 60 trading days. They believe that the high-volume return premium is a compensation for differential risk levels across stocks since higher volume implies greater divergence in investor opinion about firm value and higher stock riskiness. Later, Lerman, Livnat, and Mendenhall (2011) also document a positive relationship between earnings announcement period trading volume and the subsequent returns. Kanile, Ozoguz, and Starks (2012) find that the high-volume return premium is evident across borders to 41 counties including almost all developed equity markets and some emerging equity markets as well.

There are quite a few researches dedicated to price (return) extremes. For example, Conrad, Hameed, and Niden (1994) use weekly data to investigate the relation between trading volume and subsequent returns to individual NASDAQ securities. They find that high volume securities experience price reversals, while low volume securities experience price continuations, which supports that past trading volume is useful in explaining the short-term return reversal pattern. Cooper (1999) uses filter rules on lagged returns and lagged volume to investigate weekly profits on large-capitalization NYSE and AMEX stocks. He finds that low-growth-in-volume securities experience greater reversals, while high-growth-in-volume securities have weaker reversals. He also finds that a security is more likely to have greater reversals if it has incurred two consecutive weeks of losses or gains. When investigating momentum investing strategy, George and Hwang (2004) recommend investors focusing on stocks that trade in the top 30\% of the prior trading range to achieve better performance. Rachev et al. (2007) also study momentum strategy and document higher profits using the cumulative return strategy on past return winners and loser. Most recently, Huddart, Lang, Yetman (2009) study the volume and price patterns around a stock's 52-week price highs and lows and document an elevated trading volume and positive excess returns following the extreme event, a piece of evident 
that is especially true for small firms. Using stocks listed on NYSE and AMEX, Driessen, Lin and Hemert (2011) find that the volatility of stock returns increases after stock price breaks through 52-week high or low, which they explain by the anchoring theory. Clearly, price (return) extremes impact asset price behavior.

Since stocks with extremely high volumes can be accompanied by extremely erratic returns - high on one day, and extremely low on the next, an observation which also holds for stocks with extremely low volumes, it would be beneficial to investigate the effects of extreme returns and extreme volumes on stock returns simultaneously. In this paper, we expand the finance literature on extreme volumes further by investigating the nature of the interactions between extreme volumes and extreme returns. It remains to be determined how extreme returns in combination with extreme volumes influence future stock returns.

\section{Data}

The daily returns, trading volumes, and capitalization data are taken from the database of the Center for Research in Security Prices (CRSP). The sample includes all of the common stocks listed on the New York Stock Exchange (NYSE) with no missing data during either the portfolio formation period or the holding period, while National Association of Securities Dealers Automated Quotations (NASDAQ) firms are not included (Note 2). The sample period is from July 2, 1962 through December 31, 2010.

\section{Methodology}

This study investigates how stock prices behave around extreme trading volumes. Trading volume is measured by daily turnover ratio, which is calculated as the ratio of number of shares traded each day to the number of shares outstanding at the end of the day, taking into account the effect of capitalization on trading volume.

To start, the time interval from July 2, 1962 to December 31, 2010 has been split into non-intersecting trading intervals of 51 trading days. The last day of the trading interval is called the formation day. The previous 1, 10, 20, 30 , or 50 days are called the formation period, while the following $1,10,20,30$, or 50 days are called the holding period. The following information is obtained on the formation day for each eligible stock: the capitalization of the stock, the extreme volume, the extreme return, and the cumulative holding period returns 1, 10, 20, 30, and 50 days before and after the formation day.

All eligible stocks traded in each interval are then assigned into three groups according to their market capitalization deciles at the end of the formation day: small, medium and large. Firms in market capitalization deciles one to five are assigned to the small capitalization group; firms in deciles six to eight are assigned to the medium group, while firms in deciles nine and ten are assigned to the large group. Analysis is then performed both on the overall sample and separately on each of the three size groups.

All eligible stocks in each size group are then ranked according to volume extremes, and assigned to three portfolios: stocks with extremely low volumes belong to portfolio X1, stocks with extremely high volumes belong to portfolio $\mathrm{X} 3$, while stocks with no extreme volumes belong to portfolio X2. On a given formation day, a stock is assigned to the extremely high volume group (X3) if its trading volume on the formation day is among the top 5 in the daily trading volumes during the formation period. An extremely low volume stock (X1) is among the bottom 5 of the daily trading volumes during that period. All other stocks are classified as non-extreme volume stocks (X2).

\subsection{Returns, Volatility, and Skewness Patterns around Extreme Volumes}

We treat the occurrence of volume extremes - low or high - as an event and compare the return, volatility, and skewness patterns before and after the event. The following information is gathered for portfolios with low volume extremes (X1) and portfolios with high volume extremes (X3) respectively: the cumulative returns before and after the events, volatility (measured by standard deviation of returns) before and after the events and skewness before and after the events.

We first focus on the return patterns. Using daily returns data, the average cumulative holding period return is calculated using the equation

$$
\operatorname{Re} t=\frac{\sum_{p=1}^{P} \frac{\sum_{i=1}^{n_{s}}\left[\prod_{t=s}^{s+k}\left(1+R_{i p t}\right)-1\right]}{n_{s}}}{P}
$$


where Ret is the average cumulative holding period return in general, while RetB_X1 and RetB_X3 represent the average cumulative return before low volume extremes (X1) and before high volume extremes (X3) respectively, and RetA_X1 and RetA_X3 represent the average cumulative return after low volume extremes (X1) and after high volume extremes (X3) respectively. In the above equation, $R_{i p t}$ is the daily return of security $i$ at time $t$ from interval $p . n_{s}$ is the number of securities traded in interval $s$, the first trading day of each holding period; $k$ is the length of the holding period in days; $P$ is the number of holding intervals.

If stocks experiencing high volume extremes tend to have a higher prior returns and higher future returns than those experiencing low volume extremes, "RetB_X1- RetB_X3" and "RetA_X1-RetA_X3" will be negative —a finding echoing the high-volume return premium. If stocks earn increasing returns after having extreme volumes, high or low, both "RetB_X1-RetA_X1" and "RetB_X3-RetA_X3" will be negative.

\begin{tabular}{cccccc}
\hline $\begin{array}{c}\text { Portfolio Return } \\
\text { Differences }\end{array}$ & Sign & $\begin{array}{c}\text { Before Low } \\
\text { Volumes }\end{array}$ & $\begin{array}{c}\text { After Low } \\
\text { Volumes }\end{array}$ & $\begin{array}{c}\text { Before High } \\
\text { Volumes }\end{array}$ & $\begin{array}{c}\text { After High } \\
\text { Volumes }\end{array}$ \\
\hline RetB_X1- RetB_X3 & Negative & Lower & ----------- & Higher & ------------ \\
RetA_X1- RetA_X3 & Negative & ----------- & Lower & ---------- & Higher \\
RetB_X1- RetA_X1 & Negative & Lower & Higher & ----------- & ----------- \\
RetB_X3- RetA_X3 & Negative & ---------- & --------- & Lower & Higher \\
\hline
\end{tabular}

A similar comparison can be made for volatility patterns around extreme volumes. Let StdB_X1 and StdB_X3 represent the average volatility before low volume extremes (X1) and before high volume extremes (X3) respectively, and StdA_X1 and StdA_X3 represent the average volatility after low volume extremes (X1) and after high volume extremes (X3) respectively. The average volatility is calculated using the standard deviation of the daily returns before/after the extreme volume event. If stocks experiencing high volume extremes tend to be more volatile than those experiencing low volume extremes, prior to or after the event, "StdB_X1-StdB_X3" and "StdA_X1-StdA_X3" will be negative. If stocks tend to become more volatile after extreme volume extremes (both extreme lows and extreme highs) than before, the results of "StdB_X1-StdA_X1" and "StdB_X3-StdA_X3" will be negative.

\begin{tabular}{cccccc}
\hline $\begin{array}{c}\text { Portfolio Volatility } \\
\text { Differences }\end{array}$ & Sign & $\begin{array}{c}\text { Before Low } \\
\text { Volumes }\end{array}$ & $\begin{array}{c}\text { After Low } \\
\text { Volumes }\end{array}$ & $\begin{array}{c}\text { Before High } \\
\text { Volumes }\end{array}$ & $\begin{array}{c}\text { After High } \\
\text { Volumes }\end{array}$ \\
\hline StdB_X1-StdB_X3 & Negative & Lower & ----------- & Higher & ------------ \\
StdA_X1-StdA_X3 & Negative & ---------- & Lower & --------- & Higher \\
StdB_X1-StdA_X1 & Negative & Lower & Higher & ---------- & ---------- \\
StdB_X3-StdA_X3 & Negative & ----------- & ----------- & Lower & Higher
\end{tabular}

Finally, we compare skewness patterns around extreme volumes. Let SkewB_X1 and SkewB_X3 represent the average skewness before low volume extremes (X1) and before high volume extremes (X3) respectively, and SkewA_X1 and SkewA_X3 represent the average skewness after low volume extremes (X1) and after high volume extremes (X3) respectively. The average skewness is calculated using the sknewness of the daily returns before/after the extreme volume events. If stocks experiencing high volume extremes tend to be more skewed than those experiencing low volume extremes, prior to or after the event, "SkewB_X1- SkewB_X3" and "SkewA_X1-SkewA_X3" will be negative. If stocks tend to become more skewed after extreme volumes (both extreme lows and extreme highs) than before, the results of "SkewB_X1-SkewA_X1" and "SkewB_X3-SkewA_X3" will be negative.

\begin{tabular}{cccccc}
\hline $\begin{array}{c}\text { Portfolio Skewness } \\
\text { Differences }\end{array}$ & Sign & $\begin{array}{c}\text { Before Low } \\
\text { Volumes }\end{array}$ & $\begin{array}{c}\text { After Low } \\
\text { Volumes }\end{array}$ & $\begin{array}{c}\text { Before High } \\
\text { Volumes }\end{array}$ & $\begin{array}{c}\text { After High } \\
\text { Volumes }\end{array}$ \\
\hline SkewB_X1-SkewB_X3 & Negative & Lower & ----------- & Higher & ------------- \\
SkewA_X1-SkewA_X3 & Negative & - ----------- & Lower & ----------- & Higher \\
SkewB_X1-SkewA_X1 & Negative & Lower & Higher & ----------- & ----------- \\
SkewB_X3-SkewA_X3 & Negative & --------- & -------- & Lower & Higher \\
\hline
\end{tabular}

Overall, the comparisons of the return, volatility, and skewness patterns before and after the volume extremes give us a more complete understanding of how volume extremes influence stock returns. 


\subsection{Interaction between Extreme Volumes and Extreme Returns}

To better understand how extreme volumes and extreme returns work together to affect future stock returns, each resulting portfolio (X1, X2, and X3) for each size group is also sub-divided into three additional groups in accordance with return extremes: extremely low return (R1), non-extreme return (R2), and extremely high return (R3). On a given formation day, a stock is classified as being of extremely high return (R3) if its return on the formation day is among the top 5 out of the daily returns during the formation period, while an extremely low return stock (R1) is among the bottom 5 of the daily returns during that period. All other stocks are classified as non-extreme return stocks (R2).

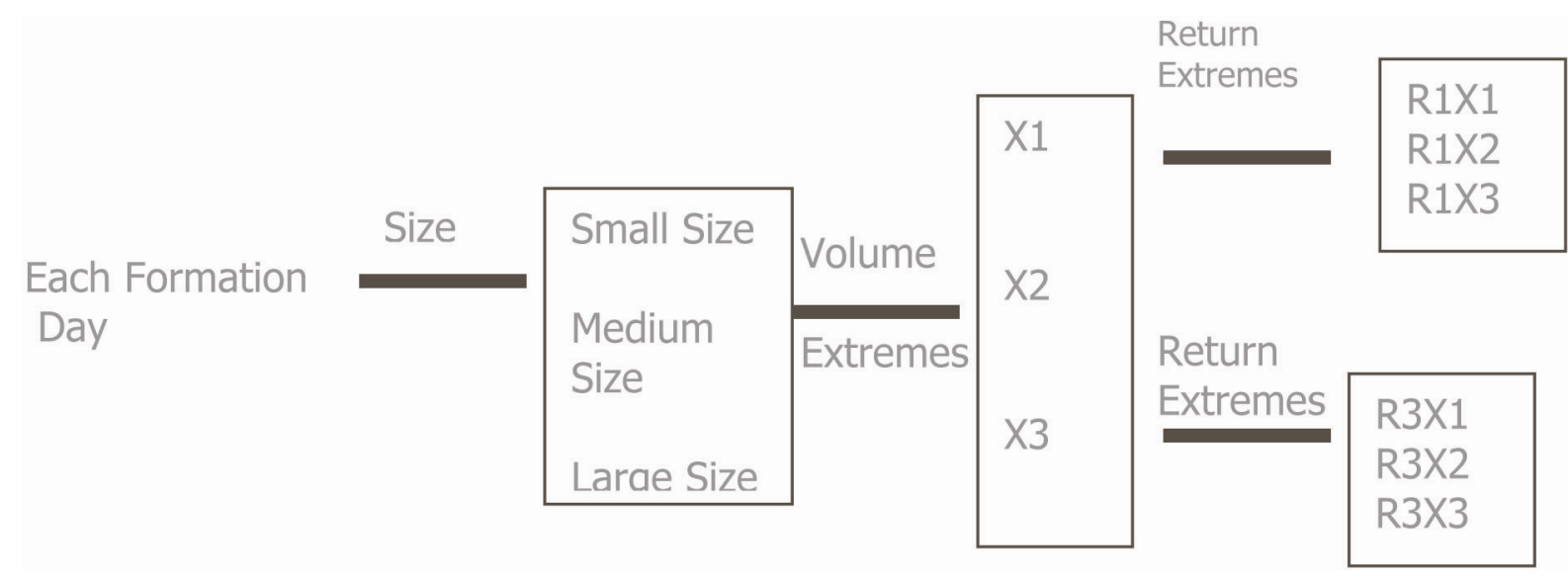

Four portfolios from each size group are of particular interest to this study-R1X1, R3X1, R1X3, and R3X3. Four net positions can then be achieved: R1X1-R1X3, R3X1-R3X3, R1X1-R3X1 and R1X3-R3X3.

A reference return strategy used by Lyon, Barber, and Tsai (1999) and Gervais, Kaniel and Mingelgrin (2001) is duplicated to calculate the average cumulative returns of these portfolios. By construction, R1X1 and R3X1 are both subject to low volume extremes but have different return extremes; R1X3 and R3X3 both have high volume extremes but have different return extremes. Observing the net average return of the net position will reveal important information about how the concurrences of return extremes with volume extremes influence future stock returns. If after controlling for the level of return extremes, stocks experiencing extremely high volume tend to perform better than those experiencing extremely low volume, the net average return of R1X1-R3X1 and R1X3-R3X3 will be significantly positive. This finding would support that the high-volume return premium exists even among those accompanied by extreme prior returns. The following table summarizes the expected results.

\begin{tabular}{ccc}
\hline & Expected Return & Reason \\
\hline R1X3-R1X1 & Positive & High-volume return premium \\
R3X3-R3X1 & Positive & High-volume return premium
\end{tabular}

If after controlling for the level of volume extremes, stocks experiencing extremely low returns tend to perform better than those experiencing extremely high returns, the net average return of R1X1-R3X1 and R1X3-R3X3 will be significantly positive. This finding would support the existence of return reversals of contrarian strategy, while the opposite would support the existence of return continuations of momentum strategy. The following table summarizes the expected results.

\begin{tabular}{lll}
\hline & Expected Return & Reason \\
\hline R1X1-R3X1 & Positive/Negative & Return Reversals/Return Continuations \\
R1X3-R3X3 & Positive/Negative & Return Reversals/Return Continuations
\end{tabular}

\section{Empirical Results}

\subsection{Returns, Volatility and Skewness Patterns around Extreme Volumes}

This section investigates the patterns of returns, volatility, and skewness before and after the occurrence of extreme volumes. The main results are presented in table 1 for overall sample and table 2 for each of the three size groups. As discussed in the methodology section, RetB_X1 and RetB_X3 represent the average cumulative return before low volume extremes (X1) and before high volume extremes (X3), and RetA_X1 and RetA_X3 represent the average 
cumulative return after low volume extremes (X1) and after high volume extremes (X3). The results for "RetB_X1RetB_X3" and "RetA_X1-RetA_X3" are both significantly negative in the overall sample and within each size group, indicating that stocks that experience extremely high volumes tend to have higher prior returns and higher future returns than those experiencing extremely low volumes. The finding that stocks with extremely high volumes yield higher future returns than those with extremely low volumes echoes the research results of Gervais, Kaniel and Mingelgrin (2001). However, since stocks with extremely high volumes could be accompanied by higher or lower prior returns, the higher future returns documented here could just purely due to return continuations from momentum strategy or return reversals from contrarian strategy. That is why we need to investigate further if the high-volume return premium still exists after controlling for the prior returns at the same level, which will be discussed in the second part of this section.

The results for "RetB_X1-RetA_X1" are significantly negative for the small size group and positive for the medium and large size groups and for the overall sample. However, the results for "RetB_X3-RetA_X3" are significantly positive throughout the overall sample and within all three size groups. Thus, we conclude that small stocks tend to earn increasing returns after extremely low volumes, while larger stocks tend to earn decreasing returns after extremely low volumes. Regardless of the size of the stock, stocks tend to earn lower returns after experiencing extremely high volumes.

Insert table $1 \& 2$ about here

Similar comparisons can be made on volatility patterns around extreme volumes. StdB_X1 and StdB_X3 represent the average volatility before low volume extremes (X1) and before high volume extremes (X3), and StdA_X1 and StdA_X3 represent the average volatility after low volume extremes (X1) and after high volume extremes (X3). The significantly positive results of "StdB_X1-StdB_X3" for the overall sample and the small size group indicate that stock returns are more volatile before extremely low volumes than before extremely high ones for small size stocks. On the contrary, the results of "StdA_X1-StdA_X3" are significantly negative for the medium and large size groups, indicating that stock returns are more volatile after extremely high volumes than after extremely low ones for larger stocks, which is consistent with the literature that a higher trading volume indicates a greater divergence of opinions, leading to more volatile stock returns.

The results of "StdB_X1-StdA_X1" are mostly positive for the overall sample and in the small and medium size group, suggesting that smaller stocks tend to become less volatile after experiencing extremely low volumes. However, the significant negative results of "StdB_X3-StdA_X3" for the overall sample and all the three size groups indicate that stocks regardless of their sizes tend to become more volatile after experiencing extremely high volumes. In sum, we find that smaller stocks experiencing extremely low volumes tend to become less volatile afterwards, while stocks of any size after experiencing extremely high volumes are found to become more volatile afterwards.

Finally, table 1 and 2 compare the skewness patterns around extreme volumes. SkewB_X1 and SkewB_X3 represent the average skewness before low volume extremes (X1) and before high volume extremes (X3), and SkewA_X1 and SkewA_X3 represent the average skewness after low volume extremes (X1) and after high volume extremes (X3). The results for "SkewB_X1-SkewB_X3" and "SkewA_X1-SkewA_X3" are significantly negative for the overall sample and within all three size groups. The negative results support the argument that stocks experiencing extremely high volume are more skewed than those experiencing extremely low volume, both before the events and after the events. The results of "SkewB_X1-SkewA_X1" are negative for the small size group and positive for the medium and large groups, indicating that small stocks tend to become more skewed after experiencing extremely low volumes, while medium and large size stocks tend to become less skewed after experiencing extremely low volumes. However, the results of "SkewB_X3-SkewA_X3" is only significantly positive for large stocks, indicating that only large stocks tend to become less skewed after extremely high volumes.

The above results can be summarized as follows: Stocks experiencing extremely high volumes generally are accompanied by higher prior and future returns when compared to stocks with extremely low volumes. Small stocks tend to earn more returns after extremely low volumes, but less returns after extremely high volumes, while larger stocks tend to earn less returns after any type of extreme volumes, high or low.

Small stock returns are more volatile before extremely low volumes than before extremely high ones. Medium and large size groups are more volatile after extremely high volumes than after extremely low ones. Small stocks tend to become less volatile after extremely low volumes, while all stocks tend to become more volatile after extremely high volumes. 
Regardless of their sizes, all stocks are more skewed before and after experiencing extremely high volumes than around extremely low volumes. Small stocks become more skewed after extreme lows, while medium and large stocks tend to be less skewed after extreme lows. Only large stocks tend to become less skewed after extremely high volumes.

\subsection{Interaction between Extreme Volumes and Extreme Returns}

In this subsection, we expand our analysis by investigating the nature of the interactions between extreme volumes and extreme returns. Given that extreme volumes in either direction may be accompanied by either extremely high returns or extremely low returns, it is justified to investigate how the extreme returns in combination with extreme volumes influence future returns.

$\mathrm{R} 1 \mathrm{X} 3-\mathrm{R} 1 \mathrm{X} 1$ is the average net return taken as a long position in extremely low return and extremely high volume stocks, and a short position in extremely low return and extremely low volume stocks. If R1X3 earns significantly higher returns than R1X1, the net average return of R1X3-R1X1 will be significantly positive. This fact is clearly observable in table 3 for the overall sample and table 4 for the small size group. This indicates that for small size stocks, the high-volume return premium exists even for those simultaneously experiencing extremely low prior returns. On the contrary, the high-volume return premium disappears for larger size stocks which simultaneously experience extremely low prior returns.

\section{Insert table $3 \& 4$ about here}

R3X3-R3X1 refers to the average net return of a long position in stocks with extremely high return and extremely high volume, and a short position in stocks with extremely high return and extremely low volume. If R3X3 earns significantly higher returns than R3X1, the net average return should be significantly positive. We only observe significantly positive returns for a holding period of 1 day. None of the results for $10,20,30$, or 50 day holding periods are significantly positive. The results in table 3 and 4 suggest that the high-volume return premium only exists for a very short time span, like a day or two, for those stocks that are accompanied by extremely high prior returns. This finding holds true regardless of size. Therefore, the high-volume return premium disappears quickly for stocks simultaneously experiencing extremely high prior returns.

$\mathrm{R} 1 \mathrm{X} 1-\mathrm{R} 3 \mathrm{X} 1$ is the average net return taken as a long position in extremely low return and extremely low volume stocks, and a short position in extremely high return and extremely low volume stocks. R1X3-R3X3 refers to the average net return of a long position in stocks with extremely low return and extremely high volume, and a short position in stocks with extremely high return and extremely high volume. Positive returns of R1X1-R3X1 and R1X3-R3X3 would lend support to the contrarian strategy where past losers outperform past winners after the effect of extreme volume is controlled, while negative returns of the above two net positions would support the momentum strategy where past winners keep being the winner. The results in table 3 and 4 are mostly neither significantly positive nor significantly negative. The only exceptions are the 10-day holding period for small size stocks and the 1-day holding period for medium size stocks, indicating return reversals for small size stocks in a 10-day holding period and return continuations for medium size stocks in a 1-day holding period. The empirical results reported here show that while accompanied by extreme volumes, past losers do not outperform past winners, and past winners do not outperform past losers neither. While there is coexistence of extreme volumes, neither return reversals nor return continuations work effectively to affect future stock returns. In other words, the existences of extreme volumes offset or cancel out the effects of return reversals or return continuations. The traditional contrarian strategy and momentum strategy therefore do not work on stocks simultaneously experiencing extreme volumes.

\section{Conclusion}

This study compares the performance of stocks with extremely high volumes directly with those experiencing extremely low volumes and gives us a more complete picture of how extreme volumes influence volatility and skewness in addition to returns. We find that the extremely high volume stocks display higher previous and future returns. Extremely high volumes tend to induce stocks of all sizes to undergo declining returns in the future. However, return patterns differ between small and larger size stocks experiencing extremely low volumes. Specifically, the returns of small stocks will increase afterwards, while the future returns of larger stocks decrease. Small stocks have less volatile returns after having extremely high volumes, and larger stocks have more volatile returns after experiencing extremely high volumes. Small stocks becomes less volatile after experiencing extremely low volumes, while larger stocks tend to become more volatile after experiencing extremely high volumes.

This study also documents that the high-volume return premium still holds strong among small size stocks which simultaneously experience extremely low prior returns. If larger size stocks simultaneously experience extremely 
low prior return, the high-volume return premium would disappear. The high-volume return premium only lasts a day and then quickly disappears among stocks simultaneously experiencing extremely high prior returns. When simultaneously accompanied by extreme trading volumes, high or low, stocks with extremely low prior returns don't outperform those with extremely high prior returns and vice versa. Therefore, this study finds no evidence to support the existence of return reversals or return continuations among stocks which simultaneously experience extreme returns and extreme volumes. The traditional contrarian strategy and momentum strategy do not profit on stocks that are simultaneously accompanied by extreme returns and extreme volumes. The existence of extreme trading volume totally cancels out any possible price impact from contrarian or momentum strategies.

\section{References}

Berkowitz, S., D. E. Logue, Noser, E. A. (1988). The total cost of transaction on the NYSE. Journal of Finance, 43 (1), 97-121. http://dx.doi.org/10.1111/j.1540-6261.1988.tb02591.x

Berkowitz, S., Logue, D.E. (2001). Transaction cost. Journal of Portfolio Management, 27 (2), 65-75. http://dx.doi.org/10.3905/jpm.2001.319793

Chordia, T., Subrahmanyam, A. (2000). Trading volume and cross-autocorrelations in stock returns. Journal of Finance, 55 (2), 913-935. http://dx.doi.org/10.3905/jpm.2001.319793

Chordia, T., Subrahmanyam A., Anshuman, V. R. (2001). Trading activity and expected stock returns. Journal of Financial Economics, 59 (1), 3-32. http://dx.doi.org/10.1016/S0304-405X(00)00080-5

Conrad J. S., Hameed, A., Niden, C. (1994). Volume and autocovariances in short horizon individual security returns. Journal of Finance, 49 (4), 1305-1329. http://dx.doi.org/10.1111/j.1540-6261.1994.tb02455.x

Cooper, M. (1999). Filter rules based on price and volume in individual security overreaction. Review of Financial Studies, 12 (4), 19-33. http://dx.doi.org/10.1093/rfs/12.4.901

Datar, V., Naik, N., Radcliffe, R. (1998). Liquidity and asset returns: an alternative test. Journal of Financial Markets, 1 (2), 203-220. http://dx.doi.org/10.1016/S1386-4181(97)00004-9

Drissen, J., Lin,T., Hemert, O. V. (2013). How the 52-week high and low affect option-implied volatilities and stock return moments. Review of Finance, 17(1), 369-401. http://dx.doi.org/10.1093/rof/rfr026

Elkins/McSherry Co. Inc, (1998). Transaction costs in Canadian and U.S. equity markets: a Study of institutional trading in cross-listed stocks. Toronto Stock Exchange.

Fama, E. F. (1998). Market efficiency, long term returns, and behavioral finance. Journal of Financial Economics, 49 (3), 286-306.

Fama, E. F. (1998). Determine the number of priced state variables in the ICAPM. Journal of Financial and Quantitative Analysis, 33 (2), 217-231. http://dx.doi.org/10.2307/2331308

Garfinkel, J. A., Sokobin, J. (2006). Volume, opinion divergence, and returns: a Study of post-earnings announcement drift. Journal of Accounting Research, 44 (1), 85-112. http://dx.doi.org/10.1111/j.1475-679X.2006.00193.x

George, T. J., Hwang, C. (2004). The 52-week high and momentum investing. Journal of Finance, 59 (5), 2145-2176. http://dx.doi.org/10.1111/j.1540-6261.2004.00695.x

Gervais, S., Kaniel, R., Mingelgrin, D. (2001). The High volume return premium. Journal of Finance, 56 (3), 877-919. http://dx.doi.org/10.1111/0022-1082.00349

Huddart, S., Lang, M., Yetman, M. H. (2008). Volume and price patterns around a stock's 52-week highs and lows: theory and evidence. Management Science, 55 (2), 16-31. http://dx.doi.org/10.1287/mnsc. 1080.0920

Kanile, R., Ozoguz, A., Starks, L. (2012). The High-volume return premium and the investor recognition hypothesis: international evidence and determinants. Journal of Financial Economics, 103 (2), 255-279.

Lerman, A., Livnat, J., Mendenhall, R. R. (2011). The High-volume return premium and post-earnings announcement drift. Working Paper

Lee, C., Swaminathan, B. (2000). Price momentum and trading volume. Journal of Finance, 55 (5), 2017-1069. http://dx.doi.org/10.1111/0022-1082.00280

Lyon, J. D., Barber, B. M., Tsai, C. (1999). Improved methods for tests of long-run abnormal stock returns. Journal of Finance, 54 (1), 165-201. http://dx.doi.org/10.1111/0022-1082.00101

MacNeil, A. J., Frey R., Embrechts, P. (2005) Quantitative risk management: Concepts, techniques and tools, Princeton University Press.

Rachev, S.T., Jašić, T., Stoyanov, S. and Fabozzi, F. J. (2007). Momentum strategies based on reward-risk stock selection stock selection criteria. Journal of Banking and Finance, 31(8), 2325-2346. http://dx.doi.org/10.1016/j.jbankfin.2007.02.006 
Sun, M. Y. (2012). High-volume return premium and liquidity premium. Journal of Business and Economic Research, forthcoming.

Wang, J. (1994). A Model of competitive stock trading volume. Journal of Political Economy, 102 (1), 127-168. http://dx.doi.org/10.1086/261924

Notes

Note 1. Sun (2012) investigates how the interaction of volume norms and volume extremes simultaneously affect future stock returns. However, our paper focuses on how the combination of extreme returns and extreme volumes predict future stock returns.

Note 2. Since dealer trades are double-counted in the NASDAQ, trading volume for NASDAQ stocks are inflated relative to NYSE and AMEX stocks.

Table 1. Comparison between return, volume and skewness patterns around extreme volumes for a 51-day interval

\begin{tabular}{|c|c|}
\hline \multicolumn{2}{|c|}{ Return Pattern } \\
\hline \multirow[t]{2}{*}{ RetB_X1-RetB_X3 } & $-0.022 * *$ \\
\hline & -15.72 \\
\hline \multirow[t]{2}{*}{ RetA_X1-RetA_X3 } & $-0.02 * *$ \\
\hline & -13.93 \\
\hline \multirow[t]{2}{*}{ RetB_X1-RetA_X1 } & $0.0050 * *$ \\
\hline & 3.70 \\
\hline \multirow[t]{2}{*}{ RetB_X3-RetA_X3 } & $0.0076^{* *}$ \\
\hline & 5.19 \\
\hline \multicolumn{2}{|c|}{ Volatility Pattern } \\
\hline \multirow[t]{2}{*}{ StdB_X1-StdB_X3 } & $0.0004 * *$ \\
\hline & 5.23 \\
\hline \multirow[t]{2}{*}{ StdA_X1-StdA_X3 } & $-3 \mathrm{E}-4$ \\
\hline & -3.32 \\
\hline \multirow[t]{2}{*}{ StdB_X1-StdA_X1 } & $0 / 0002 * *$ \\
\hline & 4.37 \\
\hline \multirow[t]{2}{*}{ StdB_X3-StdA_X3 } & $-52 \mathrm{E}-5 * *$ \\
\hline & -10.75 \\
\hline \multicolumn{2}{|c|}{ Skewness Pattern } \\
\hline \multirow[t]{2}{*}{ SkewB_X1-SkewB_X3 } & $-81 \mathrm{E}-5^{* *}$ \\
\hline & -9.36 \\
\hline \multirow[t]{2}{*}{ SkewA_X1-SkewA_X3 } & $-69 \mathrm{E}-5 * *$ \\
\hline & -8.15 \\
\hline \multirow[t]{2}{*}{ SkewB_X1-SkewA_X1 } & $348 \mathrm{E}-7$ \\
\hline & 0.46 \\
\hline \multirow[t]{2}{*}{ SkewB_X3-SkewA_X3 } & 0.0002 \\
\hline & 1.64 \\
\hline
\end{tabular}

The return, volatility, and skewness patterns are compared in this table. RetB: cumulative returns before the events. RetA: cumulative returns after the events. StdB: average volatility calculated by the standard deviation of daily returns before the events. StdA: average volatility calculated by the standard deviation of daily returns after the events. SkewB: average skewness calculated by the sknewness of daily returns before the events. SkewA: average skewness calculated by the sknewness of daily returns after the events. X1: stocks with extremely low volume. X3: stocks with extremely high volume. The numbers in the second line are t-statistics. $* *$ are significant at $1 \%$ and $*$ are significant at $5 \%$. 
Table 2. Comparison between return, volume and skewness patterns around extreme volumes for small, medium and large size groups

\begin{tabular}{|c|c|c|c|}
\hline & Small & Medium & Large \\
\hline \multicolumn{4}{|c|}{ Return Pattern } \\
\hline \multirow[t]{2}{*}{ RetB_X1-RetB_X3 } & $-0.033 * *$ & $-0.018 * *$ & $-0.007 * *$ \\
\hline & -13.59 & -7.97 & -2.81 \\
\hline \multirow[t]{2}{*}{ RetA_X1-RetA_X3 } & $-0.0238 * *$ & $-0.018 * *$ & $-0.014 * *$ \\
\hline & -9.55 & -8.21 & -6.42 \\
\hline \multirow[t]{2}{*}{ RetB_X1-RetA_X1 } & $-0.0005 *$ & $0.0104 * *$ & $0.0181 * *$ \\
\hline & -2.36 & 4.89 & 7.88 \\
\hline \multirow[t]{2}{*}{ RetB_X3-RetA_X3 } & 0.0047 & $0.0098 * *$ & $0.0102 * *$ \\
\hline & 1.86 & 4.21 & 4.22 \\
\hline \multicolumn{4}{|c|}{ Volatility Pattern } \\
\hline \multirow[t]{2}{*}{ StdB_X1-StdB_X3 } & $0.0008 * *$ & 0.0002 & $-3 \mathrm{E}-5$ \\
\hline & 5.73 & 1.32 & -0.22 \\
\hline \multirow[t]{2}{*}{ StdA_X1-StdA_X3 } & $-17 \mathrm{E}-5$ & $-46 \mathrm{E}-5 * *$ & $-51 \mathrm{E}-5^{* *}$ \\
\hline & -1.13 & -3.39 & -3.36 \\
\hline \multirow[t]{2}{*}{ StdB_X1-StdA_X1 } & $0.0003 * *$ & $0.0002 *$ & $377 \mathrm{E}-7$ \\
\hline & 3.91 & 2.34 & 0.42 \\
\hline \multirow[t]{2}{*}{ StdB_X3-StdA_X3 } & $-62 \mathrm{E}-5 * *$ & $-44 \mathrm{E}-5 * *$ & $-45 \mathrm{E}-5 * *$ \\
\hline & -7.51 & -5.82 & -5.21 \\
\hline \multicolumn{4}{|c|}{ Skewness Pattern } \\
\hline \multirow[t]{2}{*}{ SkewB_X1-SkewB_X3 } & $-0.001 * *$ & $-82 \mathrm{E}-5 * *$ & $-37 \mathrm{E}-5^{*}$ \\
\hline & -7.58 & -5.36 & -2.08 \\
\hline \multirow[t]{2}{*}{ SkewA_X1-SkewA_X3 } & $-7 \mathrm{E}-4 * *$ & $-8 \mathrm{E}-4 * *$ & $-49 \mathrm{E}-5^{* *}$ \\
\hline & -5.45 & -5.28 & -2.99 \\
\hline \multirow[t]{2}{*}{ SkewB_X1-SkewA_X1 } & $-45 \mathrm{E}-5 * *$ & $0.0003 *$ & $0.0006^{* *}$ \\
\hline & -3.95 & 2.19 & 4.07 \\
\hline \multirow[t]{2}{*}{ SkewB_X3-SkewA_X3 } & $-15 \mathrm{E}-5$ & 0.0003 & $0.0005 * *$ \\
\hline & -1.05 & 1.81 & 2.93 \\
\hline
\end{tabular}

The return, volatility, and skewness patterns are compared in this table. RetB: cumulative returns before the events. RetA: cumulative returns after the events. StdB: average volatility calculated by the standard deviation of daily returns before the events. StdA: average volatility calculated by the standard deviation of daily returns after the events. SkewB: average skewness calculated by the sknewness of daily returns before the events. SkewA: average skewness calculated by the sknewness of daily returns after the events. X1: stocks with extremely low volume. X3: stocks with extremely high volume. The numbers in the second line are t-statistics. $* *$ are significant at $1 \%$ and $*$ are significant at $5 \%$. 
Table 3. Average cumulative returns of portfolios based on extreme returns and extreme volumes for a 51-day interval

\begin{tabular}{cccccc}
\hline $\begin{array}{l}\text { formation-holding } \\
\text { period }\end{array}$ & $51-1$ & $51-10$ & $51-20$ & $51-30$ & $51-50$ \\
\hline R1X3-R1X1 & $0.0008^{* *}$ & $0.0045^{* *}$ & $0.0052^{* *}$ & $0.0049^{* *}$ & $0.0038^{*}$ \\
& 2.66 & 5.36 & 4.60 & 3.55 & 2.10 \\
R3X3-R3X1 & $0.0019^{* *}$ & 0.0009 & $0.0023^{*}$ & $0.0042^{* *}$ & $0.0044^{* *}$ \\
& 6.82 & 1.31 & 2.22 & 3.35 & 2.63 \\
R1X1-R3X1 & 0.0004 & -0.0014 & -0.0024 & -0.0011 & 0.0005 \\
& 1.25 & -1.34 & -1.58 & -0.62 & 0.22 \\
R1X3-R3X3 & $-0.0008^{* *}$ & $0.0014^{*}$ & 0.0006 & -0.0006 & -0.0009 \\
& -3.19 & 2.21 & 0.68 & -0.59 & -0.69 \\
\hline
\end{tabular}

In each trading interval, stocks are classified according to size, return extremes and volume extremes on the formation day. Size groups are based on the firm's market capitalization at the day prior to each formation period. The return extremes classification is based on whether the stock's return at the formation day is among the top or bottom 5 of the daily returns in the whole formation period. The volume extremes classification is based on whether the stock's trading volume at the formation day is among the top or bottom 5 of the daily volumes in the whole formation period. R1X1: stocks with extremely low return and extremely low volume. R3X1: stocks with extremely high return and extremely low volume. R1X3: stocks with extremely low return and extremely high volume. R3X3: stocks with extremely high return and extremely high volume. In all cases, average cumulative returns are calculated for the following different holding periods: 1, 10, 20, 30, and 50 trading days. The average cumulative returns are displayed in the first line. The numbers in the second line are t-statistics. $* *$ are significant at $1 \%$ and $*$ are significant at $5 \%$. 
Table 4. Average cumulative returns of portfolios based on extreme returns and extreme volumes for a 51-day interval for small, medium, and large size groups

\begin{tabular}{|c|c|c|c|c|c|}
\hline $\begin{array}{l}\text { formation-holding } \\
\text { period }\end{array}$ & $51-1$ & $51-10$ & $51-20$ & $51-30$ & $51-50$ \\
\hline \multicolumn{6}{|c|}{ Small Size Group } \\
\hline \multirow[t]{2}{*}{ R1X3-R1X1 } & $0.0014 *$ & $0.0078 * *$ & $0.0089 * *$ & $0.0093 * *$ & $0.0073 *$ \\
\hline & 2.49 & 5.31 & 4.52 & 3.80 & 2.30 \\
\hline \multirow[t]{2}{*}{ R3X3-R3X1 } & $0.0023 * *$ & 0.0007 & 0.0021 & $0.0043 *$ & 0.0025 \\
\hline & 4.66 & 0.61 & 1.17 & 2.03 & 0.91 \\
\hline \multirow[t]{2}{*}{ R1X1-R3X1 } & 0.0004 & -0.0026 & $-0.0054^{*}$ & -0.0023 & -0.0003 \\
\hline & 0.67 & -1.39 & -2.00 & -0.73 & -0.08 \\
\hline \multirow[t]{2}{*}{ R1X3-R3X3 } & -0.0008 & $0.0028 * *$ & 0.0017 & 0.0010 & 0.0017 \\
\hline & -1.85 & 2.61 & 1.16 & 0.55 & 0.72 \\
\hline \multicolumn{6}{|c|}{ Medium Size Group } \\
\hline \multirow[t]{2}{*}{ R1X3-R1X1 } & 0.0002 & $0.0032 *$ & 0.0028 & 0.0033 & 0.0024 \\
\hline & 0.50 & 2.29 & 1.55 & 1.58 & 0.87 \\
\hline \multirow[t]{2}{*}{ R3X3-R3X1 } & $0.0018 * *$ & 0.0010 & 0.0013 & 0.0031 & 0.0050 \\
\hline & 4.02 & 0.89 & 0.84 & 1.55 & 1.79 \\
\hline \multirow[t]{2}{*}{ R1X1-R3X1 } & 0.0004 & -0.0005 & 0.0003 & 0.0018 & 0.0055 \\
\hline & 0.89 & -0.32 & 0.14 & 0.64 & 1.45 \\
\hline \multirow[t]{2}{*}{ R1X3-R3X3 } & $-0.0010^{* *}$ & 0.0008 & 0.0006 & 0.0001 & -0.0007 \\
\hline & -2.58 & 0.84 & 0.48 & 0.07 & -0.32 \\
\hline \multicolumn{6}{|c|}{ Large Size Group } \\
\hline \multirow[t]{2}{*}{ R1X3-R1X1 } & 0.0006 & 0.0015 & 0.0024 & 0.0006 & 0.0010 \\
\hline & 1.38 & 1.16 & 1.36 & 0.28 & 0.37 \\
\hline \multirow[t]{2}{*}{ R3X3-R3X1 } & $0.0011^{*}$ & 0.0003 & 0.0020 & 0.0037 & $0.0051^{*}$ \\
\hline & 2.51 & 0.33 & 1.30 & 1.92 & 2.03 \\
\hline \multirow[t]{2}{*}{ R1X1-R3X1 } & 0.0006 & -0.0001 & -0.0005 & -0.0015 & -0.0044 \\
\hline & 1.10 & -0.08 & -0.23 & -0.55 & -1.14 \\
\hline \multirow[t]{2}{*}{ R1X3-R3X3 } & -0.0001 & 0.0005 & -0.0001 & -0.0024 & -0.0038 \\
\hline & -0.47 & 0.58 & -0.06 & -1.46 & -1.78 \\
\hline
\end{tabular}

In each trading interval, stocks are classified according to size, return extremes and volume extremes on the formation day. Size groups are based on the firm's market capitalization at the day prior to each formation period. The return extremes classification is based on whether the stock's return at the formation day is among the top or bottom 5 of the daily returns in the whole formation period. The volume extremes classification is based on whether the stock's trading volume at the formation day is among the top or bottom 5 of the daily R3X1: stocks with extremely high return and extremely low volume. R1X3: stocks with extremely low return and extremely high volume. R3X3: stocks with extremely high return and extremely high volume. In all cases, average cumulative returns are calculated for the following different holding periods: 1, 10, 20, 30, and 50 trading days. The average cumulative returns are displayed in the first line. The numbers in the second line are t-statistics. ** are significant at $1 \%$ and $*$ are significant at $5 \%$. 